\title{
Computer simulations and modeling in oncology: methods and applications
}

\author{
C. Guiot ${ }^{1}$, P. Paolo Delsanto ${ }^{2}$ \& A. S. Gliozzi ${ }^{2}$ \\ ${ }^{1}$ Department of Neuroscience, Università di Torino, Torino, Italy \\ ${ }^{2}$ Department of Physics, Politecnico di Torino, Torino, Italy
}

\begin{abstract}
Computational models and simulations can be powerful tools for gaining an insight into the extremely complex mechanisms governing tumoral growth. In order to be relied upon, however, they must be validated by comparison with sufficiently long strings of experimental or observational data. For obvious ethical reasons it is virtually impossible to obtain such data "in vivo". It may be, therefore, expedient to study the growth of tumoral lines "in vitro" or "ex vivo", i.e. by transplanting them into lab animals (e.g., mice). In fact, experiments with as many as 900 successive transplants into new healthy mice have been performed. Using a recently proposed technique for the analysis of experimental datasets (the Phenomenological Universalities Approach), we have succeeded to reproduce, to an excellent level of reliability, the results of such "multipassage" growth and to explain quantitatively why the growth curves become progressively steeper at each new transplant. We believe that our method could also be applied to study metastatic diffusion and suggest new experiments to further validate our approach and results.
\end{abstract}

Keywords: tumor models, growth models, data analysis.

\section{Introduction}

Computational models can be very useful in many subfields of biomedicine and, in particular, in oncology, due to the extreme complexity of the mechanisms governing tumoral growth, such as angiogenesis, invasion of the surrounding tissues, metastatic diffusion, etc. In fact, such models allow theoretical understanding of the processes involved, by varying the details of the proposed model or their parameters, or by adding new ingredients and/or eliminating ineffectual ones. If a satisfactory agreement is found with the experimental or observed phenomenology, the models may help to reach a good comprehension 
of the neoplastic development. As a corollary, it becomes possible to perform virtual experiments of selected therapies and to predict or optimize the outcome of suggested therapeutic protocols. In some cases they can also reduce the need of much more expensive and objectionable experiments on lab animals.

The current relevance of mathematical and computational modeling is due to a combination of related factors [1]. Among them: the advent of systemsbiology-driven concepts in biomedicine that draw from an ever increasing volume of molecular data [2-5], the introduction of novel and cancer-focused interdisciplinary funding programs at the NIH (such as the Integrative Cancer Biology Program [6]) and the decreasing cost of the computational power necessary to run large and clinically relevant simulations.

Physical models, based on a comparison with well known phenomena, which present formal analogies with some aspects of the tumoral development, may also be extremely useful, since they may suggest new mechanisms to be tested and analysed. This "crossfertilization" can be efficiently achieved by means of the Phenomenological Universalities (PUN) approach, recently proposed by Delsanto et al. [7-9]. As an example of physical models, we wish to mention here the study of tumor invasiveness, based on the analogy with two well known physical mechanisms, i.e. the mechanical insertion of a solid inclusion in an elastic material specimen or the impinging of a water drop on a solid surface [10].

Finally, biological models are essential to validate the results obtained by means of the theoretical models, both for what concerns the understanding of the phenomenology and the applications for diagnostic and/or therapeutic purposes. They include the implementation of selected tumor lines in "in vitro" or "exvivo" experiments on lab animals, such as mice.

For a broad list of recent articles and other information on this topic, we refer to the repository of mathematical models and corresponding computational codes assembled within the framework of the Center for the Development of a Virtual Tumor (CViT) Project (http://www.cvit.org), belonging to the US NIH-NCI ICBP (integrative Cancer Biology Program) [6].

There exists a large number of computational models and simulation techniques, such as cellular automata, finite difference methods, LISA (Local Interaction Simulation Approach), etc. [11-16]. They generally consist of "mesoscopic" formulations that help us to connect the macroscopic and microscopic points of view, i.e. what is mainly of clinical interest from what can be learned from the bio-chemo-physics of the cells, e.g. by means of "ab initio" calculations [17]. Such an understanding is necessary not only to predict the emergence of macroscopic phenomena out of microscopic laws, but also to correlate microscopic and macroscopic parameters [18,19].

\section{The PUN approach}

The PUN approach consists of the search of best fitting functions based only on the experimental datasets available and without any reference to the field of application. The most important PUN classes studied to date are the above- 
mentioned classes UN, which at the first level $(\mathrm{N}=0)$ correspond to unrestricted exponential growth. At the level $\mathrm{N}=1$, they yield the Gompertz law. Finally, at the level $\mathrm{N}=2$ (i.e. U2), they successfully predict the fractal properties of the solution of the growth equation at larger times.

From a purely applicative point of view, PUNs can be described as a tool for solving the following problem: let us assume that we have an experimental dataset: $y_{i}=y\left(t_{i}\right)$, where $\mathrm{t}$ can be the time (or any other independent variable) and $y$ any observable depending on it. The usual procedure is to perform a fitting of the data, but the choice of the fitting function is generally arbitrary. As a result, the analysis is, in general, only of qualitative value, and often based on the visual inspection of the plots. By contrast, we wish to proceed here in a way that is justified in the framework of a "universal" approach, i.e., totally independent of the field of application.

If the nature of the problem suggests that it can be reduced to a first order ODE, we aim to analyse it starting from the nonlinear growth equation:

$$
\dot{y}=a(y, t) y(t)
$$

where $\dot{y}=\frac{d y}{d t}$ and $a$ represent the growth rate.

Equation (1) is, however, not limited to the modelling of growth problems, since there is no restriction on the nature of the variables $y$ and $t$. Equation (1) in its complete generality cannot take us too far. In order to use it for a quantitative analysis, it is necessary to restrict its generality by means of some "constraint", which, although arbitrary, at least are independent of the particular field of application. Let us then assume that $a$ is a function solely of $z=\ln (y)$ and that its derivative with respect to $z$ may be expanded as a set of powers of $a$, i.e.

$$
b=\dot{a}=b(a)=\frac{d a}{d z} \dot{z}=a \frac{d a}{d z}=\sum_{n=1}^{\infty} \alpha_{n} a^{n}
$$

If a satisfactory fit of the experimental data is obtained by truncating the set at the N-th term (or power of $a$ ), then we state that the underlying phenomenology belongs to the Universality Class UN. It can be easily shown that the Universality Class $\mathrm{U} 1$, i.e. with $\mathrm{N}=1$ and $b=\alpha_{1} a$, represents the well-known 'Gompertz' law, which has been used for more than a century to study all kinds of growth phenomena. The class U2 includes, besides Gompertz as a special case, all the growth models proposed to date in all fields of research, i.e., besides the already mentioned model of West et al. [20,7], also the exponential, logistic, thetalogistic, potential, von Bertalanffy, etc. (see, for a review, Ref. [21]).

By solving the differential equations $\dot{z}=a$ and $\dot{a}=b$, with $b$ written, for brevity, in the case $\mathrm{N}=2$ 


$$
b=\alpha a+\beta a^{2}
$$

we find the $\mathrm{U} 2$ solution

$$
y=\left[1+\frac{\beta}{\alpha}\left(1-e^{\alpha t}\right)\right]^{-\frac{1}{\beta}}
$$

It is interesting to observe that Eq.(4) can be written as

$$
u=c_{1}+c_{2} \tau
$$

which shows that the scaling invariance, which was lost due to the nonlinearity of $a(z)$, may be recovered if the fractal-dimensioned variable $u=y^{-\beta}$ and $\tau=\exp (\alpha t)$ are considered. In fact is, in general, non-integer. In Eq. (5) $c_{1}$ and $c_{2}$ are constants: $c_{2}=-\frac{\beta}{\alpha}, c_{1}=1-c_{2}$.

It may also be useful to note that $y$ is the solution of the ODE:

$$
\dot{y}=\gamma_{1} y^{p}-\gamma_{2} y,
$$

where $p=1+\beta . \gamma_{1}$ and $\gamma_{2}$ are two constants: $\gamma_{2}=\alpha / \beta$ and $\gamma_{1}=1-\gamma_{2}$. Their sum is equal to 1 , due to the chosen normalization $y(0)=1$. Equation (6) coincides with West's universal growth equation [20], except that here $p$ may be totally general, while West and collaborators adopt Kleiber's prescription $(p=3 / 4)[22]$, which seems to be well supported by animal growth data. For other systems different choices of $p$ may be preferable: in particular C. Guiot et al. suggest a dynamical evolution of $p$ in the transition from an avascular phase to an angiogenetic stage in tumors [23].

Equation (6) has a very simple energy balance interpretation, with $\gamma_{1} y^{p}$ representing the input energy (through a fractal branched network), $\gamma_{2} y$ the metabolism and $\dot{y}$ the asymptotically vanishing growth. In fact all UN's (at least up to $\mathrm{N}=3$ ) correspond to energy conservation (or, equivalently, to the first Principle of Thermodynamics). However, in U1 there is no fractal dimensionality and both the input energy and metabolism are proportional to $\mathrm{y}$. In $\mathrm{U} 2$, as we have seen, there is fractal dimensionality in the energy input term. In U3 there is also a fractal dimensionality term (with the same exponent $p$ ) in the growth. 
Another very important property of complex systems is cyclicity, which seems to be an almost unavoidable consequence of the feedback system from the surrounding environment, particularly in biomedical and socio-economical sciences. In order to include it in the treatment, we must also consider the case of dependence of $a$ on both $\mathrm{z}$ and $\mathrm{t}$ [24]. We assume that

$$
a(z, t)=\bar{a}(z)+\tilde{a}(t)
$$

in which $a$ is assumed to be the sum of two contributions to the growth rate, one $(\bar{a}(z))$ which depends only on $z$ (or $y$ ) and the other $(\tilde{a}(t))$ solely timedependent. Then, by writing,

it follows

$$
y=\bar{y}(t) \tilde{y}(t)
$$

$$
\dot{y}=(\bar{a}+\tilde{a}) \bar{y} \tilde{y}
$$

which shows that Eq.(1) can be split into a system of two uncoupled equations

$$
\dot{\bar{y}}=\bar{a}(z) \bar{y}
$$

and

$$
\dot{\tilde{y}}=\tilde{a}(t) \tilde{y}
$$

Eq. (7) can be solved as before (for the case $a(z)$ ), giving rise to the classes UN. For the solution of Eq.(8) we can assume

$$
\tilde{a}=\dot{\tilde{z}}=\sum_{n=1}^{\infty} A_{n} E_{n}
$$

where $E_{n}=\exp \left[i\left(n \omega t+\Psi_{n}\right)\right]$.

Then, if the sum in Eq.(3) can be truncated to the M-th term, we will state that the corresponding phenomenology belongs to the class UN/TM.

\section{PUNs and the multicellular tumor spheroids (MTS) growth}

A convenient experimental tool that captures some of the most relevant features of tumor growth kinetics while allowing for a manageable description are the multicellular tumor spheroids (MTS) [17,19]. MTS are spherical aggregations of tumor cells that may be grown under strictly controlled conditions. Their simple 
geometries and the ability to produce them in large quantities has led to interesting new insights into cancer research.

In order to understand the "basics" of tumor evolution, we recall that it is generally assumed that tumors originate from a "seed" and grow by cell duplication, therefore following in a first phase an exponential growth law. As long as no mechanical or nutritional restrictions apply, they go on replicating with a constant duplication time. After a while, however, host and other constraints force the development of a necrotic core, and growth slows down towards some asymptotic level of saturation. This behaviour is well described by the well-known Gompertz law, which has been heuristically used for more than a century in biology and other disciplines. Most aggressive "in vivo" or "ex-vivo" tumors overcome nutrients deprivation by means of angiogenesis, and the neovascular network partly supports growth, as discussed by Delsanto et al. [25], following the model of West et al. [20] and West and Brown 22]. This third phase is complemented by the processes of tumor invasion and metastasis.

In Fig. 1 three regions may be well identified. In the first one, corresponding to the PUN class U0, there is an almost perfect exponential growth without necrotic core formation. In the second one, corresponding to U1, a bending of the growth curve towards some asymptotic level of saturation can be clearly observed: it is due to the decreasing availability of nutrients for the growing MTS. In the third phase (U2), a further decrease in the growth rate occurs, since the MTS approaches the borders of the culture medium in a non-uniform fashion, giving rise to some fractal structures at its surface and/or core interface.
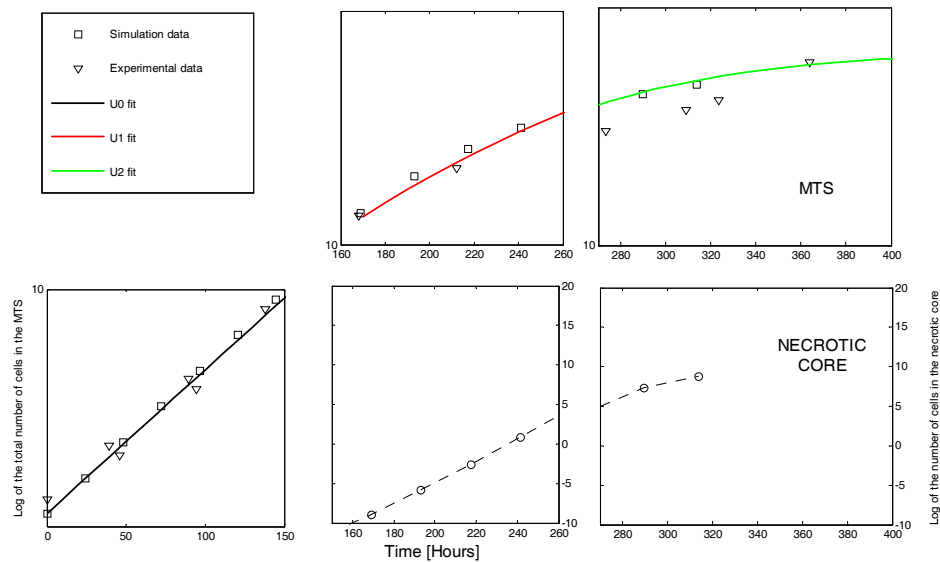

Figure 1: The three phases of growth of MTS. Temporal evolution of a MTS made of EMT6/Ro mouse mammary carcinoma cells grown in a confined culture medium. The experimental data (triangles) are taken from [26]. The "squares" and "circles" correspond to the total numbers of MTS cells and necrotic cells, respectively. They have been obtained from a mesoscopic simulation, based on the model of Delsanto et al. $[18,19]$. 


\section{PUNs and the multipassaged tumors in mice.}

By applying the PUN approach to the data of Steel [27] and McCredie and Sunderland [28], we have found that the class U2 describes extremely well both their datasets, although with accelerated time scales. If we transplant a tumor seed in a mouse flank and, after it has grown for a few days, we collect a small fraction $\mathrm{m}_{0}$ of the tumor and reimplanted it to another mouse etc. each time only for a short time $\mathrm{T}$ (e.g. ten days) tumor is allowed to grow; then we can assume at each 'passage' $n$ an experimental growth law with approximately the same rate $a$ (since free growth, in a healthy and nutrient-rich tissue is always occurring, and can write at time $t=n T+\Delta t$. In fact, experiments with as many as 900 successive transplants into new healthy mice have been performed. It was observed that tumors grow rate apparently increases at successive transplant (see Fig 2(a)). Actually, Eq.(10) shows that at each transplant the exponential trend is corrected by a term which accounts for the real age of the tumor which, by increasing at each transplant, thus accelerates the growth. In other words, the growth rate $a$ remains the same, provided the time is properly renormalized (see Fig 2(b)).

$$
m(t)=m_{0}(1+\exp (n a T)(\exp (a \Delta t)-1))
$$

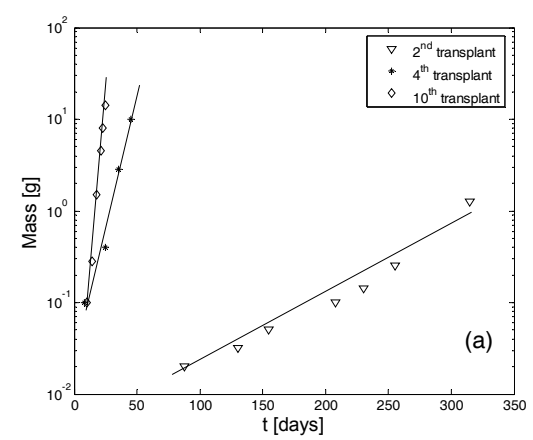

(a)

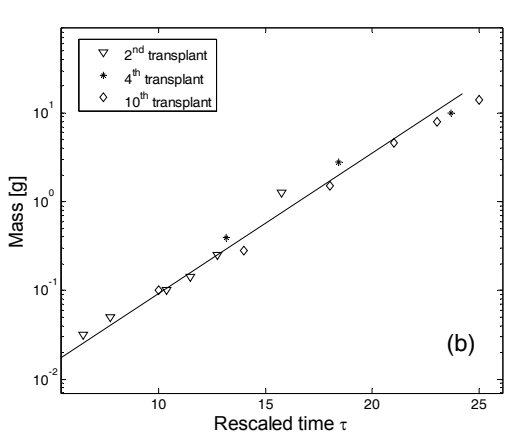

(b)

Figure 2: Results from Steel [27]. Up to 10 transplants of cells from the tumoral line rat fibroadenoma have been performed, but the curves corresponding to only three of them have been reported [9] in logarithmic scale. As discussed in the text, the averaged growth curves become increasingly steeper with successive passages, due to the aging of the newly transplanted tumor cells. By rescaling the time, we obtain a plot (Fig.2b), in which all the curves are collapsed into a unique one. 


\section{References}

[1] Deisboeck TS et al. (2009) In silico cancer modeling:is it ready for prime time. Nature Clinical Practice 6: 34

[2] Liu ET et al. (2006) In the pursuit of complexity: systems medicine in cancer biology. Cancer Cell 9: 245-247

[3] Kitano H (2002) Computational systems biology. Nature 420: 206-210

[4] Hornberg JJ et al. (2006) Cancer: a systems biology disease. Biosystems 83: $81-90$

[5] Coffey DS (1998) Self-organization, complexity and chaos: the new biology for medicine. Nat Med 4: 882-885

[6] Deisboeck TS et al. (2007) Advancing cancer systems biology: introducing the Center for the Development of a Virtual Tumor, CViT. Cancer Informatics: $1-8$

[7] Delsanto PP, ed., Universality of Nonclassical Nonlinearity with applications to NDE and Ultrasonics (Springer, 2007).

[8] Castorina P, Delsanto PP, and Guiot C. Classification Scheme for Phenomenological Universalities in Growth Problems in Physics and Other Sciences, Phys. Rev. Lett. 2006, 96:188701.

[9] Delsanto PP, Guiot C and Gliozzi AS. Scaling, growth and cyclicity in biology: a new computational approach, Theor. Biol. Med. Modell. 2008, 5:5.

[10] Guiot C, Pugno N, Delsanto PP and Desiboeck TS. Physical aspect of cancer invasion, Phys. Biol 2007,4:1-6.

[11] Capogrosso Sansone B, Delsanto P, Magnano M, Scalerandi M. (2001). Effects of anatomical constraints on tumor growth. Phys. Rev. E, vol. 64; p. 021903, ISSN: 1063-651X

[12] Scalerandi M, Pescarmona GP, Delsanto P, Capogrosso Sansone B. (2001). A LISA Model of the Response of the Vascular System to Metabolic Changes of the Cells Behavior. Phys. Rev. E, vol. 63; p. 11901, ISSN: 1063-651X

[13] Delsanto P, Romano A, Scalerandi M, Pescarmona GP. (2000). Analysis of a Phase Transition Between Tumor Growth and Latency. Phys. Rev. E, vol. 62; p. 2547-2554, ISSN: 1063-651X

[14] Scalerandi M, Romano A, Pescarmona GP, Delsanto P, Condat CA. (1999). Nutrient Competition as a Determinant for Cancer Growth. Phys. Rev. E, vol. 59; p. 2206-17, ISSN: 1063-651X

[15] Delsanto P, Gliozzi AS, Guiot C. (2008). Scaling, growth and ciclicity in biology: a new computational approach. Theor. Biol. and Med. Mod., vol. 5; p. 5, ISSN: 1742-4682

[16] Delsanto P, Gliozzi AS, Bruno CLE, Pugno N, Carpinteri A. (2008). Scaling laws and fractality in the framework of a phenomenological approach. Chaos, solitons \& fractals, ISSN: 0960-0779

[17] Chignola R, Del Fabbro A, Dalla Pellegrina C and Milotti E, Ab initio phenomenological simulation of the growth of large tumor cell populations, Phys. Biol. 2007, 4:114-33. 
[18] Delsanto P, Griffa M, Condat CA, Delsanto S, Morra L. (2005). Bridging the gap between mesoscopic and macroscopic models: the case of multicellular tumor spheroids. Phys. Rev. Lett., vol. 94; p. 148105, ISSN: 0031-9007.

[19] Delsanto P, Condat CA, Pugno N, Gliozzi AS, Griffa M (2008). A Multilevel Approach to Cancer Growth Modeling. J. Theor. Biol., vol. 250; p. 16-24, ISSN: 0022-5193.

[20] West GB, Brown JH and Enquist BJ. A general model for ontogenetic growth. Nature 2001, 413:628-631

[21] De Vladar HP, Density-Dependence as a Size-Independent Regulatory Mechanism, J. Theor. Biol., 238, 245-256 (2006).

[22] West GB and Brown JH. Life's universal scaling laws. Physics Today 2004, 57(9): 36-43; Savage VM, Deeds EJ, Fontana W. Sizing Up Allometric Scaling Theory. PLoS Comput Biol 2008, 4(9): e1000171.

[23] Guiot C, Delsanto PP, Carpinteri A, Pugno N, Mansury Y, Deisboeck TS, The dynamic evolution of the power exponent in a universal growth model of tumors, J Theor Biol (2006), 240:459-463.

[24] Delsanto PP, Gliozzi AS, and Guiot C, Scaling, Growth and Cyclicity in Biology: a New Computational Approach, Theoretical Biology and Medical Modelling (2008), 5:5.

[25] Delsanto PP, Guiot C, Degiorgis PG, Condat AC, Mansury Y and Desiboeck TS. Growth model for multicellular tumor spheroids. Appl. Phys. Lett. 2004, 85: 4225-4227.

[26] Freyer JP and Sutherland RM. Regulation of growth saturation and development of necrosis in EMT6/Ro multicellular spheroids by the glucose and oxygen supply, Cancer Res. 1986, 46:3504.

[27] Steel GG. Growth Kinetics of Tumors, Clarendon Press, Oxford, 1977.

[28] McCredie JA, Sutherland RM. Differences in growth and morphology between the spontaneous $\mathrm{C} 3 \mathrm{H}$ mammary carcinoma in the mouse and its syngeneic transplants. Cancer 1971, 27:635-642. 